\title{
Increased Cortical Porosity in Older Men With Fracture
}

\author{
Daniel Sundh, ${ }^{1,2 *}$ Dan Mellström, ${ }^{1,2 *}$ Martin Nilsson, ${ }^{1,2}$ Magnus Karlsson, ${ }^{3}$ Claes Ohlsson, ${ }^{2}$ \\ and Mattias Lorentzon ${ }^{1,2}$

\begin{abstract}
${ }^{1}$ Geriatric Medicine, Department of Internal Medicine and Clinical Nutrition, Institute of Medicine, University of Gothenburg, Gothenburg, Sweden
${ }^{2}$ Center for Bone Research at the Sahlgrenska Academy, Institute of Medicine, University of Gothenburg, Gothenburg, Sweden

${ }^{3}$ Clinical and Molecular Osteoporosis Research Unit, Department of Clinical Sciences, Lund University, and Department of Orthopaedics, Skåne

University Hospital, Malmö, Sweden
\end{abstract}

\begin{abstract}
Cortical porosity increases with age and affects bone strength, but its association with fracture in older men is unknown. The aim of this study was to investigate whether cortical porosity is associated with prevalent fractures in older men. A subsample of 456 men aged $80.2 \pm 3.5$ (mean \pm SD) years, with available high-resolution peripheral quantitative computed tomography measurements at the tibia from the 5-year follow-up exam, was drawn from the prospective MrOS Gothenburg study. Dual-energy X-ray absorptiometry was used to measure areal bone mineral density (aBMD). Data on physical activity, calcium intake, medications, diseases, and smoking were collected on questionnaires at the follow-up exam. Of 87 men (19.1\%) with fracture at or after age 50 years (all fracture group), 52 (11.4\%) had had a self-reported fracture before the baseline exam and 35 (7.7\%) had had an X-ray-verified fracture between baseline and follow-up. Men in the all-fracture group and in the X-ray-verified group had $15.8 \%(13.2 \% \pm 4.9 \%$ versus $11.4 \% \pm 3.8 \% ; p<0.001)$ and $21.6 \%(14.1 \% \pm 5.2 \%$ versus $11.6 \% \pm 3.9 \% ; p<0.01)$ higher cortical porosity, respectively, than men in the nonfracture group. The independent associations between bone microstructure parameters and fracture were tested using multivariate logistic regression with age, height, weight, calcium intake, smoking, physical activity, medications, and diseases as covariates. Cortical porosity was independently associated with any fracture (reported or X-ray-verified; OR per SD increase 1.49; $95 \%$ confidence interval (Cl), 1.17 to 1.90 ) and with any X-ray-verified fracture alone (OR $1.73 ; 95 \% \mathrm{Cl}, 1.23$ to 2.42 ). Including aBMD (spine or hip, respectively) in the multivariate logistic regression above revealed that cortical porosity was associated with any fracture $(\mathrm{OR} 1.54 ; 95 \% \mathrm{Cl}, 1.17$ to 2.01$)$ and with X-ray-verified fracture alone (OR $1.49 ; 95 \% \mathrm{Cl}, 1.00$ to 2.22 ). Cortical porosity was associated with prevalence of fracture even after adjustment for aBMD. (c) 2015 The Authors. Journal of Bone and Mineral Research published by Wiley Periodicals, Inc. on behalf of American Society for Bone and Mineral Research (ASBMR).
\end{abstract}

KEY WORDS: CORTICAL POROSITY; FRACTURE; MEN; BONE MINERAL DENSITY; OSTEOPOROSIS

\section{Introduction}

$\mathrm{O}$ steoporosis is characterized by low bone mineral density (BMD) and impaired bone microarchitecture, leading to decreased bone strength and increased risk of fracture. ${ }^{(1)}$ Measurement of areal bone mineral density (aBMD) by dualenergy X-ray absorptiometry (DXA) is a well-established method to identify people with osteoporosis and increased risk for fracture; $^{(2)}$ however, only one in five men sustaining a nonvertebral fracture are diagnosed with osteoporosis through DXA-derived aBMD. ${ }^{(3)}$ Other factors such as poor balance and risk of falling are therefore likely to play an important role. Furthermore, aBMD does not provide information about the bone microstructure and geometry that could better reflect bone strength.
Osteoporosis has not been as well studied in men as in women. One-third of osteoporotic fractures occur in men, ${ }^{(4)}$ and their lifetime risk for a future fracture is considerable. After the age of 50 years, approximately $13 \%$ of all men will sustain an osteoporotic fracture. ${ }^{(5)}$ Gathering information about the microarchitecture of the bone was previously only possible through invasive examinations. Noninvasive high-resolution peripheral quantitative computed tomography (HR-pQCT) now allows assessment of the bone microstructure at peripheral sites such as the radius and tibia. ${ }^{(6)}$ Women and men with vertebral fractures have been reported to have altered trabecular microstructure and cortical bone geometry, ${ }^{(7-9)}$ and reduced trabecular bone density was also observed in women with prior wrist fracture. ${ }^{(10,11)}$ Earlier studies using HR-pQCT have shown that measuring the trabecular microstructure provides better

This is an open access article under the terms of the Creative Commons Attribution-NonCommercial-NoDerivs License, which permits use and distribution in any medium, provided the original work is properly cited, the use is non-commercial and no modifications or adaptations are made.

Received in original form October 7, 2014; revised form March 10, 2015; accepted March 11, 2015. Accepted manuscript online March $16,2015$.

Address correspondence to: Mattias Lorentzon, MD, Geriatric Medicine, Building K, 6th Floor, Sahlgrenska University Hospital, Mölndal, 43180 Mölndal, Sweden. E-mail: Mattias.Lorentzon@medic.gu.se

Additional Supporting Information may be found in the online version of this article.

*DS and DM contributed equally to this work.

Journal of Bone and Mineral Research, Vol. 30, No. 9, September 2015, pp 1692-1700

DOI: $10.1002 / j b m r .2509$

() 2015 The Authors. Journal of Bone and Mineral Research published by Wiley Periodicals, Inc. on behalf of American Society for Bone and Mineral Research (ASBMR). 
discrimination between postmenopausal women with and without fractures than measuring aBMD in the hip using DXA. ${ }^{(7)}$

Bone loss in older ages has been reported to be mainly cortical, and associated with declining serum estradiol levels, ${ }^{(12)}$ because of larger accessible areas for bone resorption in cortical versus trabecular bone. This results in cortical remnants and lower bone strength. ${ }^{(13)}$ Cortical porosity has been found to be a main structural component of the cortex and therefore an important factor in bone strength. ${ }^{(14)}$ With a nominal isotropic resolution of $82 \mu \mathrm{m}$ it is possible to separate cortical from trabecular bone and to perform a more advanced analysis to obtain more detailed information about cortical geometry, density, and porosity, although smaller cortical pores cannot be detected. Whether cortical porosity can be used to distinguish older men with prevalent fracture from men without fracture is as yet unknown.

The aim of this study was to investigate whether older men with prevalent fracture (reported or X-ray-verified) had increased cortical porosity than men without fracture, and whether this association is independent of aBMD.

\section{Subjects and Methods}

\section{Study subjects}

The MrOS study is a prospective multicenter study including 3014 elderly men from Hong Kong, the United States, and Sweden. ${ }^{(15)}$ This study comprises a subsample of men included in the Gothenburg $(n=1010)$ part of the Swedish cohort. ${ }^{(16,17)}$ At baseline, letters were sent to a randomly selected group of subjects (men aged 69 to 81 years old) identified in national population registers and contacted by telephone. To be eligible for the study, the participants had to be able to walk without aid, sign an informed consent, and complete a questionnaire. The inclusion rate at baseline for the Swedish part of the MrOS study was $45 \% .{ }^{(18)}$

In total, 600 of the original 1010 study subjects from the Gothenburg cohort participated in the 5-year follow-up exam, leading to a $59.4 \%$ inclusion rate. Of these, 456 subjects (mean age $80.2 \pm 3.5$ years) were able to perform all steps of the present study. The participants were younger (age 74.5 \pm 3.0 years versus $75.9 \pm 3.2$ years, $p<0.001)$ than nonparticipants, but similar in height $(176 \pm 64 \mathrm{~cm}$ versus $175 \pm 64 \mathrm{~cm}, p>0.05)$ and weight $(81.1 \pm 11 \mathrm{~kg}$ versus $80.9 \pm 13 \mathrm{~kg}, p>0.05)$. The proportion of men with self-reported fractures at baseline did not differ between these groups (included 147/456 versus not included $172 / 554, p=0.68$ using $\chi^{2}$ test).

Anthropometrics were measured on standardized equipment and the average of two consecutive height measurements was calculated. If the two measurements differed by $\geq 5 \mathrm{~mm}$ a third measurement was performed and the two most similar measurements were used to calculate the average. A standardized questionnaire was used to collect information about smoking habits, calcium intake, medical history (eg, stroke, rheumatoid arthritis, and Parkinson's disease), medications, and physical activity. Current physical activity was assessed on a selfreported questionnaire. ${ }^{(19)}$ Current physical activity was assessed using the Physical Activity Scale for the Elderly (PASE), a validated self-reported questionnaire designed to measure physical activity in individuals aged 65 years or older. ${ }^{(20)}$ This scale comprises 12 items about physical activity over a 7-day period prior to the assessment. The PASE score was computed by multiplying the amount of time spent (hours per week) or participation (yes/no) in different activities by empirically derived weights and summing the products for all 12 items.
Daily intake of calcium was calculated from the questionnaire items about calcium-containing foods (eg, diary products, vegetables). The baseline questionnaire was also used to gather information about self-reported fracture sustained from the age of 50 years; X-ray-verified fractures from the time of the baseline exam to the date of follow-up were collected from patient records. All fractures were defined and categorized by site only as peripheral (upper and lower arm and leg), osteoporotic (hip, vertebrae, wrist, and humerus), or all fractures (excluding hand, finger, foot, toe, and skull). No information was available on amount of trauma. Two groups of fractures were analyzed: all identified fractures (self-reported and X-ray-verified fractures) and $X$-ray-verified fractures only. Men in the different fracture categories (eg, peripheral, osteoporotic, and multiple fractures) were always compared with men without fracture. Vertebral fractures extracted from the baseline questionnaire were defined only as yes/no. Vertebral fractures in the X-ray-verified fracture group were all clinical fractures, defined by a radiologist, identified using radiology reports from patient records made between the baseline and follow-up exam.

The ethical review board in Gothenburg approved the study and all participants signed an informed consent.

\section{Assessment of BMD}

aBMD $\left(\mathrm{g} / \mathrm{cm}^{2}\right)$ was measured at the hip, femoral neck, lumbar spine $\left(\mathrm{L}_{1}-\mathrm{L}_{4}\right)$, total body, and left arm on a Hologic QDR 4500/ADelphi DXA (Hologic, Waltham, MA, USA). Left arm aBMD, including the complete arm, was taken from the total body scan. All measurements were performed using the same DXA device. The coefficient of variation (CV) for these measurements ranged from $0.5 \%$ to $3.0 \%$.

\section{Assessment of bone microarchitecture}

Volumetric BMD (vBMD) and bone microstructure were investigated at the distal tibia with a high-resolution 3D peripheral quantitative computed tomography (HR-pQCT) device (XtremeCT; Scanco Medical AG, Brüttisellen, Switzerland) using a protocol as described. ${ }^{(21)}$ The operator placed a reference line at the distal articular plateau and captured the first slice at $22.5 \mathrm{~mm}$ proximal to that line. A total of 110 parallel images with an isotropic resolution of $82 \mu \mathrm{m}$ were obtained, resulting in a 3D representation of a $9.02 \mathrm{~mm}$ section of tibia. The total time was approximately $3 \mathrm{~min}$ and the effective dose generated was about $3 \mu$ Sv.

After processing the images as described, ${ }^{(22)}$ the following parameters were obtained: cortical thickness (Ct.Th, $\mathrm{mm}$ ), cortical cross-sectional area (CSA, $\mathrm{mm}^{2}$ ), cortical vBMD (vBMD, $\mathrm{mg} / \mathrm{cm}^{3}$ ), trabecular bone volume fraction (BV/TV, \%), trabecular number $\left(\mathrm{mm}^{-1}\right)$, trabecular thickness (Tb.Th, $\mathrm{mm}$ ), and trabecular separation (Tb.Sp, $\mathrm{mm}$ ). The CVs ranged from $0.1 \%$ to $1.6 \%$ for tibia measurements. Of the 600 men enrolled in the follow-up study, 478 were measured with the HR-pQCT. Image quality was graded on a scale as recommended by the manufacturer (Scanco Medical AG), ranging from grade 1 (highest quality) to grade 5 (unacceptable). Of the 478 images, four were excluded due to misplaced scout views and 18 because of low quality.

\section{Cortical evaluation}

Images were processed by a customized version of the manufacturer's Image Processing Language (IPL v5.08b; Scanco Medical AG) according to a previously described method. ${ }^{(23)} \mathrm{A}$ 
contour was automatically placed around the bone to delineate the periosteal surface from extra-osseal soft tissue. Another endosteal contour was automatically placed to separate trabecular from cortical bone. All contours for both segmentation steps were carefully inspected and areas where they were misplaced were manually corrected. If the automated algorithm included, for example, soft tissue within the bone section in the first proposed contour, the operator corrected the mistake. When all contours were in order, cortical porosity was defined within the contours, excluding artifacts such as surface roughness and transcortical foramen or erosions. In the end, the segmented and the cortical porosity images were combined to create a more defined cortical compartment. Parameters obtained from this method were cortical pore diameter (Ct.Po.Dm), cortical pore volume (Ct.Po.V), and cortical bone volume (Ct.BV). Using this segmentation process, cortical porosity (Ct.Po, \%) could be calculated by the following formula: ${ }^{(23,24)}$

$$
\text { Ct.Po }(\%)=\text { Ct.Po.V } /(\text { Ct.Po.V + Ct.BV })
$$

The CV for porosity and mean cortical pore diameter was $5.5 \%$ and $3.9 \%$, respectively, for tibia.

\section{Statistical analysis}

Differences in the bone variables and covariates between men with and without fractures were compared using an independent sample $t$ test for continuous variables and $\chi^{2}$ for categorical variables (percentage). Bivariate logistic regression models were used to test the crude association between bone traits and fractures and described as odds ratios (OR) with $95 \%$ confidence intervals $(\mathrm{Cl})$. Multivariate logistic regression models were performed to evaluate the independence of covariates (smoking, physical activity, daily intake of calcium, height, weight, age, per os [po] glucocorticoids, and diseases) and calculate the adjusted OR. $\chi^{2}$ Test was used to investigate whether the association between cortical porosity and fracture prevalence was linear or if there were signs of a threshold for this association by comparing the proportion of men with fracture in the different quartiles of cortical porosity. To evaluate whether cortical porosity was independently associated with fracture, the above models were also adjusted for aBMD. The most strongly associated aBMD site (highest OR) for a specific fracture type was used as the aBMD covariate. Values of $p<0.05$ were considered significant and all analyses were performed using SPSS (Version 20, SPSS, Inc, Chicago, IL, USA).

\section{Results}

The average time for participants from age 50 years to the follow-up study was $30.2 \pm 3.5$ years. Of the 456 men (age $80.2 \pm 3.5$ years, mean \pm SD) enrolled in this follow-up, 87 had a self-reported or X-ray-verified prevalent fracture. Of 87 men $(19.1 \%)$ with fracture at or after age 50 years (all fracture group), 52 (11.4\%) had had a self-reported fracture before the baseline exam, and 35 (7.7\%) had had an X-ray-verified fracture between baseline and follow-up ( $5.7 \pm 0.8$ years). Of these 87 men, 52 had an osteoporotic fracture and 34 had a peripheral fracture, 29 men had two or more fractures, and 369 men had no previous fracture. Of the 35 men with X-ray-verified fracture, 28 had an osteoporotic, 15 had a peripheral, and 9 had more than one fracture. The fractures in each category are described in Table 1 (X-ray-verified fractures) and Supporting Table 1 (all fractures).

\section{Anthropometrics and environmental factors}

All identified fractures (including self-reported and X-ray-verified)

Men with any, osteoporotic only, and multiple fractures weighed less than men with no fracture; men with osteoporotic fracture were older than men without fracture; and men with multiple fractures had a higher proportion of prior stroke (Supporting Table 2).

\section{X-ray-verified fractures}

Men with any, peripheral, and osteoporotic X-ray-verified fracture weighed less than men in the nonfracture reference group (Table 2). Men with any X-ray-verified fracture were less physically active and men with multiple fractures consumed more calcium than the nonfractured subjects (Table 2).

\section{aBMD}

All identified fractures (including self-reported and X-ray-verified)

Men with any identified fracture had lower unadjusted aBMD at the total hip $\left(0.89 \pm 0.16\right.$ versus $\left.0.97 \pm 0.14 \mathrm{~g} / \mathrm{cm}^{2} ; 8.3 \%\right)$, femoral neck $\left(0.73 \pm 0.13\right.$ versus $\left.0.79 \pm 0.13 \mathrm{~g} / \mathrm{cm}^{2} ; 7.6 \%\right)$, lumbar spine $\left(0.99 \pm 0.15\right.$ versus $\left.1.09 \pm 0.19 \mathrm{~g} / \mathrm{cm}^{2} ; 9.2 \%\right)$, and

Table 1. Number of Each Type of Fracture Within the X-ray-Verified Fracture Group

\begin{tabular}{lcccc}
\hline Place of fracture & All fractures & Peripheral fracture & Osteoporotic fracture \\
\hline Hip & One: 3 & One: 3 & One: 3 & One: 8 \\
Wrist and lower arm & One: 8 & One: 8 & One: 17 & Fractures \\
Vertebral & One: 11 & - & Two: 4 & One: 1 \\
& Two: 4 & - & One: 4 & Two: 4 \\
Shoulder and clavicular & One: 3 & One: 4 & - & One: 2 \\
Humerus & One: 4 & - & - & - \\
Rib, sternum, and thorax & One: 8 & - & - & - \\
Femur & - & - & - & - \\
Patella & - & - & -
\end{tabular}

Each place and category of fracture is reported as the number of fractures per individual: number of individuals with one or two fractures. 
Table 2. Characteristics of Men With Only X-ray-Verified Fractures Versus Men With No Fracture

\begin{tabular}{|c|c|c|c|c|c|}
\hline & $\begin{array}{c}\text { No } \\
\text { fracture }\end{array}$ & $\begin{array}{c}\text { All } \\
\text { fractures }\end{array}$ & $\begin{array}{l}\text { Peripheral } \\
\text { fracture }\end{array}$ & $\begin{array}{l}\text { Osteoporotic } \\
\text { fracture }\end{array}$ & $\begin{array}{c}\quad \geq 2 \\
\text { Fractures }\end{array}$ \\
\hline Subjects $(n)$ & 421 & 35 & 15 & 28 & 9 \\
\hline Age (years) & $80.1 \pm 3.5$ & $81.0 \pm 3.6$ & $81.0 \pm 3.6$ & $81.3 \pm 3.1$ & $80.1 \pm 3.2$ \\
\hline Height $(\mathrm{cm})$ & $175.1 \pm 6.5$ & $174.1 \pm 5.4$ & $174.0 \pm 5.1$ & $173.7 \pm 4.9$ & $173.2 \pm 5.5$ \\
\hline Weight (kg) & $79.8 \pm 11.2$ & $75.3 \pm 11.0^{\mathrm{a}}$ & $73.6 \pm 10.1^{\mathrm{a}}$ & $73.1 \pm 9.0^{\mathrm{b}}$ & $75.8 \pm 12.1$ \\
\hline Calcium intake (mg/day) & $930 \pm 424$ & $1067 \pm 503$ & $951 \pm 370$ & $1086 \pm 491$ & $1290 \pm 607^{\mathrm{a}}$ \\
\hline Smoking, $n(\%)$ & $17(4.0)$ & $3(8.6)$ & $1(6.7)$ & $3(10.7)$ & - \\
\hline Glucocorticoids p.o., $n$ (\%) & $12(2.9)$ & $1(2.9)$ & - & - & - \\
\hline Secondary osteoporosis/fall inducing disease, $n(\%)$ & $49(11.7)$ & $6(17.1)$ & $2(13.3)$ & $3(10.7)$ & $2(22.2)$ \\
\hline Prior stroke, $n(\%)$ & $39(9.3)$ & $6(17.1)$ & $2(13.3)$ & $3(10.7)$ & $2(22.2)$ \\
\hline Parkinson's disease, $n$ (\%) & $4(1.0)$ & - & - & - & - \\
\hline Rheumatoid arthritis, $n$ (\%) & $12(2.9)$ & - & - & - & - \\
\hline Current physical activity, PASE score & $393 \pm 227$ & $313 \pm 219^{a}$ & $327 \pm 182$ & $331 \pm 226$ & $418 \pm 348$ \\
\hline
\end{tabular}

Age, height, weight, calcium intake, and current physical activity are presented by mean \pm SD. Differences between the various fracture groups and the reference group (no fracture group) were tested by independent samples $t$ test. Differences in proportions were tested by $\chi^{2}$.

PASE $=$ Physical Activity Scale for the Elderly.

${ }^{a} \mathrm{p}<0.05$.

$b_{p}<0.01$.

left arm aBMD $\left(0.82 \pm 0.13\right.$ versus $\left.0.87 \pm 0.13 \mathrm{~g} / \mathrm{cm}^{2} ; 5.8 \%\right)$ than men without fracture (Supporting Table 3 ). These differences were more pronounced between men with prior osteoporotic fracture and men without fracture.

\section{$X$-ray-verified fractures}

Men with any X-ray-verified fracture had lower unadjusted aBMD at the total hip (13.5\%), femoral neck (12.8\%), lumbar spine $(12.0 \%)$, and left arm aBMD (5.8\%) than men without a fracture (Table 3). Similar significant differences at the spine and total hip were observed between men with X-ray-verified peripheral and osteoporotic fracture and men without a fracture (Table 3).

\section{Bone microstructure and prevalent fracture}

All identified fractures (including self-reported and X-ray-verified)

At the distal tibia men who had sustained any fracture had lower unadjusted trabecular bone volume fraction (14.3 \pm 3.0 versus $15.1 \pm 2.7 ; 5.3 \%)$, cortical $\mathrm{vBMD}(747 \pm 92.0$ versus $\left.786 \pm 68.3 \mathrm{mg} / \mathrm{cm}^{3} ; 5.0 \%\right)$, cortical thickness $(0.88 \pm 0.34$ versus $1.01 \pm 0.30 \mathrm{~mm} ; 12.9 \%)$, and higher cortical porosity $(13.2 \% \pm$ $4.9 \%$ versus $11.4 \% \pm 3.8 \% ; 15.8 \%)$ than men without a fracture

Table 3. aBMD, Geometry, and Microstructure in Men With Only X-ray-Verified Fractures Versus Men With No Fracture

\begin{tabular}{|c|c|c|c|c|c|}
\hline & No fracture & All fractures & Peripheral fracture & Osteoporotic fracture & $\geq 2$ Fractures \\
\hline \multicolumn{6}{|l|}{ DXA } \\
\hline Subjects $(n)$ & 392 & 30 & 13 & 25 & 7 \\
\hline Total hip aBMD $\left(\mathrm{g} / \mathrm{cm}^{2}\right)$ & $0.96 \pm 0.14$ & $0.83 \pm 0.18^{c}$ & $0.86 \pm 0.20^{a}$ & $0.80 \pm 0.16^{c}$ & $0.91 \pm 0.23$ \\
\hline Femoral neck aBMD $\left(\mathrm{g} / \mathrm{cm}^{2}\right)$ & $0.78 \pm 0.13$ & $0.68 \pm 0.15^{c}$ & $0.72 \pm 0.16$ & $0.67 \pm 0.13^{c}$ & $0.76 \pm 0.18$ \\
\hline Lumbar spine aBMD $\left(\mathrm{g} / \mathrm{cm}^{2}\right)$ & $1.08 \pm 0.18$ & $0.95 \pm 0.18^{c}$ & $0.97 \pm 0.18^{a}$ & $0.95 \pm 0.16^{c}$ & $0.97 \pm 0.24$ \\
\hline Left arm aBMD $\left(\mathrm{g} / \mathrm{cm}^{2}\right)$ & $0.86 \pm 0.13$ & $0.81 \pm 0.19^{\mathrm{a}}$ & $0.78 \pm 0.10^{\mathrm{a}}$ & $0.81 \pm 0.20$ & $0.84 \pm 0.12$ \\
\hline \multicolumn{6}{|l|}{ HR-pQCT } \\
\hline Subjects $(n)$ & 421 & 35 & 15 & 28 & 9 \\
\hline Trabecular BV/TV (\%) & $15.0 \pm 2.7$ & $13.2 \pm 3.0^{c}$ & $14.3 \pm 3.0$ & $13.3 \pm 3.1^{\mathrm{b}}$ & $14.3 \pm 3.6$ \\
\hline Trabecular number $\left(\mathrm{mm}^{-1}\right)$ & $1.98 \pm 0.30$ & $1.82 \pm 0.32^{\mathrm{b}}$ & $1.82 \pm 0.21^{\mathrm{a}}$ & $1.81 \pm 0.32^{\mathrm{b}}$ & $1.83 \pm 0.47$ \\
\hline Trabecular thickness (mm) & $0.08 \pm 0.01$ & $0.07 \pm 0.01$ & $0.08 \pm 0.02$ & $0.07 \pm 0.01$ & $0.08 \pm 0.02$ \\
\hline Trabecular separation (mm) & $0.44 \pm 0.08$ & $0.49 \pm 0.10^{c}$ & $0.48 \pm 0.06$ & $0.49 \pm 0.10^{c}$ & $0.50 \pm 0.15$ \\
\hline Cortical thickness (mm) & $1.01 \pm 0.30$ & $0.78 \pm 0.34^{c}$ & $0.86 \pm 0.35$ & $0.79 \pm 0.35^{c}$ & $0.81 \pm 0.36$ \\
\hline Cortical porosity (\%) & $11.6 \pm 3.9$ & $14.1 \pm 5.2^{\mathrm{b}}$ & $15.2 \pm 5.9^{\mathrm{a}}$ & $13.9 \pm 5.3^{b}$ & $13.6 \pm 6.2$ \\
\hline Cortical pore diameter (mm) & $0.21 \pm 0.02$ & $0.22 \pm 0.03$ & $0.23 \pm 0.03^{b}$ & $0.21 \pm 0.03$ & $0.22 \pm 0.03$ \\
\hline Cortical vBMD $\left(\mathrm{mg} / \mathrm{cm}^{3}\right)$ & $784 \pm 69.3$ & $712 \pm 104^{c}$ & $718 \pm 123$ & $707 \pm 107^{c}$ & $731 \pm 87.5^{\mathrm{a}}$ \\
\hline
\end{tabular}

Unadjusted bone values are given as mean \pm SD. Differences between the various fracture groups and the reference group (no fracture group) were tested by independent samples $t$ test.

${ }^{a} \mathrm{p}<0.05$

${ }^{b} p<0.01$.

${ }^{c} \mathrm{p}<0.001$. 
(Supporting Table 3). Men with peripheral fractures had reduced cortical vBMD $\left(735 \pm 96.0\right.$ versus $\left.786 \pm 68.3 \mathrm{mg} / \mathrm{cm}^{3} ; 6.5 \%\right)$, cortical thickness $(0.85 \pm 0.32$ versus $1.01 \pm 0.30 \mathrm{~mm} ; 15.8 \%)$, and substantially increased cortical porosity $(14.1 \% \pm 4.8 \%$ versus $11.4 \% \pm 3.8 \% ; 23.7 \%$ ) at the distal tibia (Supporting Table 3).

\section{$X$-ray-verified fractures}

The 35 men with any X-ray-verified fracture during the study had lower trabecular bone volume fraction (12.0\%), cortical vBMD (9.2\%), cortical thickness (22.8\%), and higher cortical porosity $(21.6 \%)$ than men without fracture (unadjusted bone parameters; Table 3). Men with X-ray-verified peripheral fracture were observed to have substantially (although not significantly $[p=0.06])$ lower cortical thickness (14.9\%), and especially increments in cortical porosity (31.0\%), than men with no fracture (unadjusted bone parameters; Table 3).

\section{Fracture prevalence by quartile of cortical porosity}

The proportion of men with fractures (self-reported or X-ray-verified) was higher in the fourth quartile of cortical porosity than in the two lowest quartiles for all (31/117 versus 17/116 [Q1] and 17/110 [Q2]), peripheral (15/95 versus 5/98 [Q1] and 4/95 [Q2]), and osteoporotic fractures (22/102 versus 8/101 [Q1] and 7/98 [Q2]; Supporting Table 4). Similar trends were observed in the analysis of X-ray-verified fractures only, but only the proportion of peripheral fractures differing significantly between cortical porosity quartiles (Table 4).

Low aBMD and impaired bone microstructure are independently associated with prevalent fracture

\section{All identified fractures (including self-reported and X-ray-verified) and $a B M D$}

In bivariate logistic regression analysis, lower aBMD was associated with increased prevalence of fracture (Supporting Table 5). After adjustments for confounders, including age, height, weight, smoking, physical activity, daily calcium intake, po glucocorticoids, and diseases, multivariate regression analysis showed that aBMD of the total hip, femoral neck, lumbar spine, and left arm was associated with all (total hip OR 1.62; $95 \% \mathrm{Cl}, 1.18$ to 2.21 ; and lumbar spine OR $1.74 ; 95 \% \mathrm{Cl}, 1.25$ to 2.42 ), peripheral, and osteoporotic fractures (Supporting Table 5). Left arm aBMD was most strongly associated with self-reported peripheral fractures (OR 4.15; $95 \% \mathrm{Cl}, 1.94$ to 8.86 per SD decrease) and with multiple fractures (OR $4.55 ; 95 \% \mathrm{Cl}, 1.78$ to 11.6 ) (Supporting Table 5).

\section{$X$-ray-verified fractures and $a B M D$}

In a multivariate regression analysis, after adjustment for cofounders, aBMD measurements of the spine and hip were significantly associated with prevalence of X-ray-verified osteoporotic and all fracture (Table 5). Left arm aBMD was associated with peripheral fractures (OR 6.37; $95 \% \mathrm{Cl}, 1.69$ to 24.0 ) but not with all, multiple, or osteoporotic fractures (Table 5).

All identified fractures (including self-reported and X-ray-verified) and bone microstructure

Multivariate regression analysis was performed with the confounders used above to investigate the association between bone microstructure parameters and fracture prevalence. Of the trabecular measurements, bone volume fraction, trabecular number, and separation were significantly associated with prevalence of osteoporotic fracture. All and multiple fractures were significantly associated with bone volume fraction (Supporting Table 6). Cortical porosity was independently associated with prevalence of all fracture (OR 1.49; $95 \% \mathrm{Cl}, 1.17$ to 1.90$)$, peripheral fracture (OR 1.93; 95\% Cl, 1.33 to 2.79), osteoporotic fracture (OR $1.85 ; 95 \% \mathrm{Cl}, 1.36$ to 2.52 ), and multiple fractures (OR 1.56; $95 \% \mathrm{Cl}, 1.06$ to 2.28; Supporting Table 6). Cortical vBMD and cortical thickness were both independently associated with all, osteoporotic, multiple, and peripheral fractures (Supporting Table 6).

\section{$X$-ray-verified fractures and bone microstructure}

Multivariate regression analysis was performed as above to investigate the association between the microstructure parameters and X-ray-verified fracture prevalence. Trabecular bone volume fraction, number, and separation were significantly associated with prevalence of all and osteoporotic X-ray-verified fracture (Table 6). Cortical porosity was independently associated with all, osteoporotic, and peripheral X-ray-verified fractures, and cortical vBMD was in dependently associated with all, osteoporotic, multiple, and peripheral X-ray-verified fractures (Table 6).

Multivariate logistic regression with cortical porosity and the most strongly associated aBMD site for a specific fracture type

\section{All identified fractures (including self-reported and X-ray-verified)}

Cortical porosity was associated independent of left arm aBMD with osteoporotic and peripheral fracture in a model with age, height, weight, smoking, physical activity, daily calcium intake, po glucocorticoids, and diseases used as additional covariates. Using the same covariates, an association independent of lumbar spine aBMD was found between cortical porosity and all fractures (Table 7).

Table 4. Number of Each Type of Fracture Within Different Quartiles of Cortical Porosity for X-ray-Verified Fractures Only

\begin{tabular}{lcccc}
\hline & Q1 & Q2 & Q3 & Q4 \\
\hline Cortical porosity (\%) & $7.05 \pm 1.30$ & $10.0 \pm 0.72$ & $12.8 \pm 0.78$ & $17.1 \pm 2.74$ \\
All fractures, $\mathrm{n}(\%)$ & $6 / 116(5.2)$ & $6 / 110(5.5)$ & $9 / 113(8.0)$ & $14 / 117(12.0)$ \\
Peripheral fracture, $\mathrm{n}(\%)$ & $3 / 111(2.7)$ & $1 / 101(1.0)$ & $3 / 105(2.9)$ & $8 / 105(7.6)^{*}$ \\
Osteoporotic fracture, $\mathrm{n}(\%)$ & $6 / 114(5.3)$ & $4 / 104(3.9)$ & $7 / 109(6.4)$ & $11 / 108(10.2)$ \\
Fractures $\geq 2, \mathrm{n}(\%)$ & $3 / 113(2.7)$ & $1 / 105(1.0)$ & $2 / 106(1.9)$ & $3 / 106(2.8)$ \\
\hline
\end{tabular}

Number of men with fracture/total number of men by quartile of cortical porosity. For each category of fracture, the total quartile number excludes men with other types of fractures.

${ }^{*} \mathrm{Q} 4>\mathrm{Q} 2$. 
Table 5. Crude and Adjusted ORs with $95 \%$ Cls Describing the Association Between DXA Variables and Prevalence of X-ray-Verified Fractures Only

\begin{tabular}{|c|c|c|c|c|}
\hline \multirow[b]{2}{*}{ DXA } & \multicolumn{2}{|c|}{ All fractures } & \multicolumn{2}{|c|}{ Peripheral fracture } \\
\hline & Crude & Adjusted & Crude & Adjusted \\
\hline Total hip aBMD $\left(\mathrm{g} / \mathrm{cm}^{2}\right)$ & $2.81(1.78-4.45)^{c}$ & $2.94(1.72-5.01)^{c}$ & $2.11(1.08-4.11)^{\mathrm{a}}$ & $2.22(1.00-4.96)$ \\
\hline Femoral neck aBMD $\left(\mathrm{g} / \mathrm{cm}^{2}\right)$ & $2.49(1.54-4.03)^{c}$ & $2.49(1.46-4.26)^{c}$ & $1.77(0.89-3.52)$ & $1.69(0.77-3.73)$ \\
\hline Lumbar spine aBMD $\left(\mathrm{g} / \mathrm{cm}^{2}\right)$ & $2.41(1.48-3.93)^{c}$ & $2.36(1.38-4.04)^{b}$ & $2.25(1.09-4.63)^{\mathrm{a}}$ & $2.03(0.88-4.71)$ \\
\hline \multirow[t]{2}{*}{ Left arm aBMD $\left(\mathrm{g} / \mathrm{cm}^{2}\right)$} & $2.16(1.17-4.00)^{\mathrm{a}}$ & $1.90(0.93-3.90)$ & $4.51(1.60-12.7)^{b}$ & $6.37(1.69-24.0)^{b}$ \\
\hline & \multicolumn{2}{|c|}{ Osteoporotic fracture } & \multicolumn{2}{|c|}{$\geq 2$ Fractures } \\
\hline DXA & Crude & Adjusted & Crude & Adjusted \\
\hline Total hip aBMD $\left(\mathrm{g} / \mathrm{cm}^{2}\right)$ & $3.61(2.11-6.17)^{c}$ & $3.36(1.82-6.21)^{c}$ & $1.51(0.67-3.41)$ & $2.05(0.72-5.86)$ \\
\hline Femoral neck aBMD $\left(\mathrm{g} / \mathrm{cm}^{2}\right)$ & $3.25(1.83-5.75)^{\mathrm{c}}$ & $2.99(1.60-5.62)^{c}$ & $1.22(0.55-2.72)$ & $1.52(0.56-4.12)$ \\
\hline Lumbar spine aBMD $\left(\mathrm{g} / \mathrm{cm}^{2}\right)$ & $2.73(1.56-4.77)^{c}$ & $2.35(1.29-4.30)^{\mathrm{b}}$ & $2.07(0.81-5.33)$ & $2.29(0.73-7.22)$ \\
\hline Left arm aBMD $\left(\mathrm{g} / \mathrm{cm}^{2}\right)$ & $2.25(1.14-4.44)^{\mathrm{a}}$ & $1.64(0.77-3.50)$ & $1.39(0.42-4.61)$ & $2.69(0.44-16.3)$ \\
\hline
\end{tabular}

Values are given as ORs with $95 \% \mathrm{Cl}$ per SD decrease. Associations were tested by logistic regression. Adjusted model included age, height, weight, physical activity, smoking, daily intake of calcium, glucocorticoids po, and secondary osteoporosis/fall inducing disease as covariates.

Crude $=$ unadjusted model.

${ }^{\mathrm{a}} p<0.05$.

${ }^{\mathrm{b}} p<0.01$.

${ }^{c} p<0.001$.

Other microstructure measurements were also tested for association with fracture when aBMD was included in the multivariate model as above. In this analysis, cortical thickness was associated independent of left arm aBMD with self-reported osteoporotic fracture (OR 1.65; $95 \% \mathrm{Cl}, 1.09$ to 2.48). Cortical vBMD was associated independently of lumbar spine and left arm aBMD with all and osteoporotic fractures (OR 1.47; $95 \% \mathrm{Cl}$, 1.10 to 1.95 and OR $1.85 ; 95 \% \mathrm{Cl}, 1.29$ to 2.65 , respectively).

\section{$X$-ray-verified fractures}

Cortical porosity was associated with all and peripheral X-ray-verified fractures independently of hip and left arm

Table 6. Crude and Adjusted ORs with 95\% Cls Describing the Association Between HR-pQCT Variables and Prevalence of X-ray-Verified Fractures Only

\begin{tabular}{|c|c|c|c|c|}
\hline \multirow[b]{2}{*}{ HR-pQCT } & \multicolumn{2}{|c|}{ All fractures } & \multicolumn{2}{|c|}{ Peripheral fracture } \\
\hline & Crude & Adjusted & Crude & Adjusted \\
\hline Trabecular BV/TV (\%) ${ }^{\mathrm{d}}$ & $2.01(1.39-2.91)^{c}$ & $1.94(1.30-2.91)^{\mathrm{b}}$ & $1.32(0.77-2.24)$ & $1.23(0.68-2.22)$ \\
\hline Trabecular number $\left(\mathrm{mm}^{-1}\right)^{\mathrm{d}}$ & $1.76(1.21-2.56)^{\mathrm{b}}$ & $1.79(1.15-2.78)^{\mathrm{b}}$ & $1.84(1.04-3.26)^{\mathrm{a}}$ & $1.68(0.85-3.33)$ \\
\hline Trabecular thickness $(\mathrm{mm})^{\mathrm{d}}$ & $1.38(0.96-1.97)$ & $1.44(0.97-2.14)$ & $0.79(0.47-1.32)$ & $0.85(0.48-1.50)$ \\
\hline Trabecular separation $(\mathrm{mm})^{\mathrm{e}}$ & $1.71(1.26-2.32)^{c}$ & $1.78(1.24-2.55)^{\mathrm{b}}$ & $1.52(0.95-2.42)$ & $1.40(0.79-2.49)$ \\
\hline Cortical thickness $(\mathrm{mm})^{\mathrm{d}}$ & $2.13(1.46-3.12)^{c}$ & $2.06(1.36-3.13)^{c}$ & $1.67(0.97-2.89)$ & $1.75(0.93-3.28)$ \\
\hline Cortical porosity (\%) ${ }^{\mathrm{e}}$ & $1.75(1.27-2.41)^{c}$ & $1.73(1.23-2.42)^{\mathrm{b}}$ & $2.25(1.39-3.63)^{c}$ & $2.36(1.38-4.04)^{b}$ \\
\hline Cortical pore diameter $(\mathrm{mm})^{\mathrm{e}}$ & $1.34(0.97-1.85)$ & $1.26(0.89-1.78)$ & $1.90(1.22-2.97)^{\mathrm{b}}$ & $1.70(1.04-2.78)^{a}$ \\
\hline \multirow[t]{2}{*}{ Cortical vBMD $\left(\mathrm{mg} / \mathrm{cm}^{3}\right)^{\mathrm{d}}$} & $2.16(1.58-2.94)^{c}$ & $2.18(1.52-3.13)^{c}$ & $1.97(1.31-2.96)^{b}$ & $2.00(1.23-3.26)^{\mathrm{b}}$ \\
\hline & \multicolumn{2}{|c|}{ Osteoporotic fracture } & \multicolumn{2}{|c|}{$\geq 2$ Fractures } \\
\hline HR-pQCT & Crude & Adjusted & Crude & Adjusted \\
\hline Trabecular BV/TV (\%) ${ }^{\mathrm{d}}$ & $1.94(1.30-2.92)^{\mathrm{b}}$ & $1.75(1.12-2.74)^{\mathrm{a}}$ & $1.32(0.67-2.60)$ & $1.36(0.62-2.94)$ \\
\hline Trabecular number $\left(\mathrm{mm}^{-1}\right)^{\mathrm{d}}$ & $1.84(1.21-2.81)^{\mathrm{b}}$ & $1.71(1.04-2.82)^{\mathrm{a}}$ & $1.68(0.84-3.39)$ & $1.37(0.57-3.31)$ \\
\hline Trabecular thickness $(\mathrm{mm})^{\mathrm{d}}$ & $1.29(0.87-1.91)$ & $1.32(0.85-2.05)$ & $0.77(0.40-1.49)$ & $1.02(0.46-2.24)$ \\
\hline Trabecular separation $(\mathrm{mm})^{\mathrm{e}}$ & $1.74(1.25-2.43)^{\mathrm{b}}$ & $1.68(1.12-2.51)^{\mathrm{a}}$ & $1.76(1.03-3.02)^{\mathrm{a}}$ & $1.70(0.85-3.39)$ \\
\hline Cortical thickness $(\mathrm{mm})^{\mathrm{d}}$ & $2.16(1.42-3.29)^{c}$ & $2.10(1.32-3.36)^{b}$ & $2.02(0.99-4.12)$ & $2.88(1.18-7.01)^{\mathrm{a}}$ \\
\hline Cortical porosity (\%) ${ }^{\mathrm{e}}$ & $1.73(1.20-2.48)^{\mathrm{b}}$ & $1.68(1.15-2.46)^{\mathrm{b}}$ & $1.60(0.88-2.91)$ & $1.62(0.85-3.10)$ \\
\hline Cortical pore diameter $(\mathrm{mm})^{\mathrm{e}}$ & $1.27(0.89-1.83)$ & $1.16(0.79-1.72)$ & $1.40(0.77-2.55)$ & $1.16(0.56-2.39)$ \\
\hline Cortical vBMD $\left(\mathrm{mg} / \mathrm{cm}^{3}\right)^{\mathrm{d}}$ & $2.26(1.61-3.19)^{c}$ & $2.29(1.55-3.40)^{c}$ & $1.90(1.08-3.34)^{\mathrm{a}}$ & $2.46(1.23-4.90)^{a}$ \\
\hline
\end{tabular}

Associations were tested by logistic regression. Adjusted model included age, height, weight, physical activity, smoking, daily intake of calcium, glucocorticoids po, and secondary osteoporosis/fall-inducing disease as covariates. Crude model is the unadjusted model.

${ }^{\mathrm{a}} p<0.05$.

${ }^{\mathrm{b}} p<0.01$.

${ }^{c} p<0.001$.

dValues are given as ORs with $95 \%$ Cls per SD decrease.

eValues are given as ORs with $95 \% \mathrm{Cls}$ per SD increase. 
Table 7. aBMD Independent Associations Between Cortical Porosity and All Fractures (Including Self-Reported Fractures and X-rayVerified) as Well as X-ray-Verified Fractures Alone

\begin{tabular}{lcc} 
HR-pQCT & All identified fractures & X-ray verified fractures \\
\hline All fractures & $1.54(1.17-2.01)^{\mathrm{b}, \mathrm{d}}$ & $1.49(1.00-2.22)^{\mathrm{a}, \mathrm{e}}$ \\
Peripheral fracture & $1.72(1.15-2.56)^{\mathrm{b}, \mathrm{f}}$ & $2.15(1.16-3.97)^{\mathrm{a}, \mathrm{f}}$ \\
Osteoporotic fracture & $1.83(1.30-2.58)^{\mathrm{c}, \mathrm{f}}$ & $1.44(0.92-2.27)^{\mathrm{e}}$ \\
Multiple fractures $(\geq 2)$ & $1.47(0.95-2.27)^{\mathrm{f}}$ & - \\
\hline
\end{tabular}

Values are given as ORs with $95 \%$ Cls per SD decrease in cortical porosity at the tibia. Associations were tested by logistic regression with aBMD, age, height, weight, physical activity, smoking, daily intake of calcium, glucocorticoids po, and secondary osteoporosis/fall-inducing disease, as covariates. The aBMD site most strongly associated with each fracture type was used each respective regression model.

${ }^{\mathrm{a}} p<0.05$.

${ }^{\mathrm{b}} p<0.01$.

${ }^{c} p<0.001$.

${ }^{\mathrm{d}}$ Lumbar spine aBMD.

eHip aBMD.

fLeft arm aBMD.

aBMD, respectively (Table 7). Cortical vBMD was also associated with all X-ray-verified fractures (OR $1.67 ; 95 \% \mathrm{Cl}, 1.07$ to 2.59 ) and osteoporotic X-ray-verified fracture (OR 1.76; $95 \% \mathrm{Cl}, 1.07$ to 2.90) independently of hip aBMD. After adjustments for aBMD, trabecular BV/TV was not associated with all or any other category of prevalent fracture.

\section{Discussion}

In this study we report the association between cortical porosity and prevalence of both self-reported and X-ray-verified fractures in older men. Increased cortical porosity was associated with higher prevalence of all, peripheral, osteoporotic, and multiple fractures. Using logistic regression analyses, we were able to demonstrate that cortical porosity was associated independently of DXA-derived aBMD with all identified and $X$-ray-verified prevalent fractures. To our knowledge, this is the first study reporting the association between cortical porosity and prevalent fracture in older men.

Cortical bone has been reported to be an important determinant of bone strength and a major contributor to fracture risk in the elderly. As Zebaze and colleagues ${ }^{(13)}$ reported, bone loss is mainly trabecular until age 65 years, followed by cortical bone loss, which occurs due to larger remodeling areas in the cortical bone. As a result, fractures affecting bone sites with mainly cortical bone (eg, femoral neck and long bones) become more common with age than fractures in the vertebrae, which contain mainly trabecular bone.

In a large sample of postmenopausal women, a great majority $(82 \%)$ of women sustaining a fragility fracture had a radius aBMD T-score of above $-2.5 .^{(25)}$ Thus, DXA-derived aBMD is not sufficient to identify patients at risk for fracture. The association between trabecular bone microstructure, assessed with HR$\mathrm{pQCT}$, and prevalence of wrist fracture in postmenopausal women has been reported earlier. Melton and colleagues ${ }^{(10)}$ described major differences in many HR-pQCT-derived trabecular parameters, especially trabecular separation and connectivity, between fractured women and controls. In their cohort, cortical VBMD and thickness differed between women with and without fractures, but no difference was found in cortical porosity of the radius. However, in a re-analysis of women from the same cohort using an alternative method (STRAX ; StraxCorp
Pty Ltd, Victoria, Australia) to obtain cortical porosity, this bone trait was useful in identifying osteopenic women at risk for fracture despite their low risk based on $\mathrm{BBMD}^{(26)}$ which seems to indicate that the use of cortical microstructural parameters could improve the assessment of fracture risk. Cortical porosity was seen to increase with the age $(r=0.57-0.58)$ of the included women (interquartile range [IQR] 56 to 71 years), ${ }^{(26)}$ which implies that cortical porosity would contribute substantially more to bone strength and be of larger clinical significance in older rather than younger patients. Cortical porosity assessed with the STRAX method is several-fold higher than previously reported in HR-pQCT studies ${ }^{(10,14)}$ and in this study. Studies investigating the role of either these methods to predict fractures are lacking.

Szulc and colleagues ${ }^{(8)}$ reported that men with vertebral fractures had poor cortical bone status, reflected by reduced thickness and vBMD. However, after adjusting for aBMD, they did not observe any associations between prevalence of a single peripheral fracture and bone microstructure traits except in a small group of men $(n=15)$ with multiple peripheral fractures who had increased trabecular spacing and distribution. Absence of significant associations for the microarchitecture parameters could be due to a relatively young population (mean age of $73 \pm 8$ years for cases and $69 \pm 9$ years for controls). Also, because none of the peripheral fractures were confirmed by $X$-ray reports, the lack of association could have been influenced by fracture misclassification, previously been reported to be as high as $7 \%$ false negative ${ }^{(27)}$ and $11 \%$ to $20 \%$ false positive. ${ }^{(27,28)}$ Another cortical bone measurement, cortical thickness, in the tibia has been associated independently of aBMD with fracture prevalence in a cohort of postmenopausal women. ${ }^{(7)}$

As in previous studies, we also observed a lower trabecular bone volume fraction in subjects with fracture than in subjects without fracture. $^{(6,29)}$ This difference was most likely due to a lower observed trabecular number. However, associations with trabecular microstructure and prevalence of fracture were no longer apparent after adjustments for aBMD. Our observed independent associations for cortical, but not trabecular, parameters could be because of our older population (age $80.2 \pm 3.5$ years), likely because of the more important role of cortical, rather than trabecular, bone at higher ages. ${ }^{(13)}$ Our analyses of fracture prevalence by quartiles of cortical porosity indicate that a nonlinear relationship between this trait and fracture could exist. The 
proportion of men with prevalent fracture was fairly similar in the two lowest quartiles and increased substantially in the fourth quartile, suggesting that porosity is only important for fracture risk when it is rather high.

Earlier dehydration or deuterium oxide experiments to measure bone water concentration have indicated a much higher cortical porosity ${ }^{(30)}$ than we observed in this study. Measurements of cortical porosity by HR-pQCT have been found to underestimate true cortical porosity since only large pores (above $130 \mu \mathrm{m}$ in diameter) are detected. ${ }^{(26)}$ Although the method used to evaluate cortical porosity in this study underestimates the actual level of porosity, it is possible that it is able at least to reflect interindividual differences in porosity. Our multivariate logistic regression models and the use of aBMD as a covariate provide novel information that cortical porosity is independently associated with fracture prevalence, indicating that this bone trait provides unique information not dependent on areal BMD.

Limitations of the present study include its cross-sectional retrospective design and the inclusion of all fractures regardless of trauma severity. Studies have reported that study subjects often have difficulty recalling a fracture event, ${ }^{(28)}$ therefore using only X-ray-verified fractures would have been desirable. However, our results for both all fractures and only $X$-ray-verified fractures were fairly similar. In most previous studies, measurements using HR-pQCT were performed on non-weight bearing bone (ie, the radius) and compared against the prevalence of wrist fracture. In contrast, we performed our analyses on a weight bearing bone, ie, the tibia, and compared all fractures and the defined subgroups of fracture. Comparison of our results with previously performed studies could therefore be difficult, because cortical porosity may differ between the two bone sites.

In conclusion, no earlier studies have examined the association between cortical porosity and the prevalence of fracture in older men. In this study we report that cortical porosity was associated with prevalence of fracture independent of aBMD and other covariates. Future prospective studies with incident fractures as an outcome variable would be important in determining the role of cortical porosity and other bone microstructure parameters in fracture prediction.

\section{Disclosures}

All authors state that they have no conflicts of interest.

\section{Acknowledgments}

This study was supported by the Swedish Research Council, the Swedish Foundation for Strategic Research, European Commission, the Lundberg Foundation, the Torsten and Ragnar Söderberg's Foundation, Petrus and Augusta Hedlund's Foundation, the ALF/LUA grant from the Sahlgrenska University Hospital, the Novo Nordisk Foundation, and Gustaf V's and Queen Victoria's Freemason Foundation.

Authors' roles: (a) Made substantial contributions to conception and design, acquisition of data, or analysis and interpretation of data: DS, ML, DM, MN, MK, and CO. (b) Participated in drafting the manuscript or revising it critically for important intellectual content: DS, ML, DM, MN, MK, and CO. (c) Approved the final version of the submitted manuscript: $D S, M L, D M, M N$, $\mathrm{MK}$, and CO. Agree to be accountable for all aspects of the work in ensuring that questions related to the accuracy or integrity of any part of the work are appropriately investigated and resolved: $\mathrm{DS}, \mathrm{ML}, \mathrm{DM}, \mathrm{MN}, \mathrm{MK}$, and CO.

\section{References}

1. [No authors listed]. Consensus development conference: prophylaxis and treatment of osteoporosis. Am J Med. 1991;90(1):107-10.

2. Kelsey JL, Browner WS, Seeley DG, Nevitt MC, Cummings SR. Risk factors for fractures of the distal forearm and proximal humerus. The Study of Osteoporotic Fractures Research Group. Am J Epidemiol. 1992;135(5):477-89.

3. Schuit SC, van der Klift M, Weel $A E$, et al. Fracture incidence and association with bone mineral density in elderly men and women: the Rotterdam Study. Bone. 2004;34(1):195-202.

4. Nguyen TV, Center JR, Eisman JA. Osteoporosis in elderly men and women: effects of dietary calcium, physical activity, and body mass index. J Bone Miner Res. 2000;15(2):322-31.

5. Melton LJ, Chrischilles EA, Cooper C, Lane AW, Riggs BL. How many women have osteoporosis? (Reprinted from Journal Bone and Mineral Research, vol 7, pg 1005, 1992). J Bone Miner Res. 2005;20(5):886-92.

6. Boutroy S, Bouxsein ML, Munoz F, Delmas PD. In vivo assessment of trabecular bone microarchitecture by high-resolution peripheral quantitative computed tomography. J Clin Endocrinol Metab. 2005;90(12):6508-15.

7. Sornay-Rendu E, Boutroy S, Munoz F, Delmas PD. Alterations of cortical and trabecular architecture are associated with fractures in postmenopausal women, partially independent of decreased BMD measured by DXA: the OFELY study. J Bone Miner Res. 2007;22(3):425-33.

8. Szulc P, Boutroy S, Vilayphiou N, Chaitou A, Delmas PD, Chapurlat R. Cross-sectional analysis of the association between fragility fractures and bone microarchitecture in older men: the STRAMBO study. J Bone Miner Res. 2011;26(6):1358-67.

9. Stein EM, Liu XS, Nickolas TL, et al. Microarchitectural abnormalities are more severe in postmenopausal women with vertebral compared to nonvertebral fractures. J Clin Endocrinol Metab. 2012;97(10):E1918-26.

10. Melton LJ 3rd, Christen D, Riggs BL, et al. Assessing forearm fracture risk in postmenopausal women. Osteoporos Int. 2010;21(7):1161-69.

11. Rozental TD, Deschamps LN, Taylor A, et al. Premenopausal women with a distal radial fracture have deteriorated trabecular bone density and morphology compared with controls without a fracture. J Bone Joint Surg Am. 2013;95(7):633-42.

12. Vandenput $L$, Lorentzon $M$, Sundh $D$, et al. Serum estradiol levels are inversely associated with cortical porosity in older men. J Clin Endocrinol Metab. 2014;99(7):E1322-6.

13. Zebaze RM, Ghasem-Zadeh A, Bohte A, et al. Intracortical remodelling and porosity in the distal radius and post-mortem femurs of women: a cross-sectional study. Lancet. 2010;375(9727):1729-36

14. Kazakia GJ, Nirody JA, Bernstein G, Sode M, Burghardt AJ, Majumdar $\mathrm{S}$. Age- and gender-related differences in cortical geometry and microstructure: improved sensitivity by regional analysis. Bone. 2013;52(2):623-31.

15. Orwoll E, Blank JB, Barrett-Connor E, et al. Design and baseline characteristics of the osteoporotic fractures in men (MrOS) study-a large observational study of the determinants of fracture in older men. Contemp Clin Trials. 2005;26(5):569-85.

16. Mellstrom $D$, Johnell $O$, Ljunggren $O$, et al. Free testosterone is an independent predictor of BMD and prevalent fractures in elderly men: MrOS Sweden. J Bone Miner Res. 2006;21(4):529-35.

17. Ohlsson C, Mellstrom D, Carlzon D, et al. Older men with low serum IGF-1 have an increased risk of incident fractures: the MrOS Sweden Study. J Bone Miner Res. 2011;26(4):865-72.

18. Mellstrom D, Vandenput $L$, Mallmin $\mathrm{H}$, et al. Older men with low serum estradiol and high serum SHBG have an increased risk of fractures. J Bone Miner Res. 2008;23(10):1552-60. 
19. Nilsson M, Sundh D, Ohlsson C, Karlsson M, Mellstrom D, Lorentzon $M$. Exercise During growth and young adulthood is independently associated with cortical bone size and strength in old Swedish men. J Bone Miner Res. 2014;29(8):1795-804.

20. Washburn RA, McAuley E, Katula J, Mihalko SL, Boileau RA. The Physical Activity Scale for the Elderly (PASE): evidence for validity. J Clin Epidemiol. 1999;52(7):643-51.

21. MacNeil JA, Boyd SK. Improved reproducibility of high-resolution peripheral quantitative computed tomography for measurement of bone quality. Med Eng Phys. 2008;30(6):792-9.

22. Laib A, Hauselmann $H J$, Ruegsegger $P$. In vivo high resolution $3 D-Q C T$ of the human forearm. Technol Health Care. 1998; 6(5-6):329-37.

23. Burghardt AJ, Buie HR, Laib A, Majumdar S, Boyd SK. Reproducibility of direct quantitative measures of cortical bone microarchitecture of the distal radius and tibia by HR-pQCT. Bone. 2010;47(3):519-28.

24. Ostertag A, Peyrin F, Fernandez S, Laredo JD, de Vernejoul MC, Chappard C. Cortical measurements of the tibia from high resolution peripheral quantitative computed tomography images: a comparison with synchrotron radiation micro-computed tomography. Bone. 2014;63:7-14.
25. Siris ES, Chen YT, Abbott TA, et al. Bone mineral density thresholds for pharmacological intervention to prevent fractures. Arch Intern Med. 2004;164(10):1108-12.

26. Bala Y, Zebaze R, Ghasem-Zadeh A, et al. Cortical porosity identifies women with osteopenia at increased risk for forearm fractures. J Bone Miner Res. 2014;29(6):1356-62.

27. Ismail AA, O'Neill TW, Cockerill W, et al. Validity of self-report of fractures: results from a prospective study in men and women across Europe. EPOS Study Group. European Prospective Osteoporosis Study Group. Osteoporos Int. 2000;11(3):248-54.

28. Nevitt MC, Cummings SR, Browner WS, et al. The accuracy of selfreport of fractures in elderly women: evidence from a prospective study. Am J Epidemiol. 1992;135(5):490-9.

29. Stein EM, Liu XS, Nickolas TL, et al. Abnormal microarchitecture and reduced stiffness at the radius and tibia in postmenopausal women with fractures. J Bone Miner Res. 2010;25(12): 2572-81.

30. Techawiboonwong A, Song HK, Leonard MB, FW Wehrli. Cortical bone water: in vivo quantification with ultrashort echo-time $M R$ imaging. Radiology. 2008;248(3):824-33. 